\title{
Multiscale Modelling of Neuronal Signalling
}

\author{
Nicolas Le Novère \\ Computational Neurobiology, EMBL-EBI, \\ Wellcome Trust Genome Campus, \\ Hinxton, Cambridge, United-Kingdom \\ lenov@ebi.ac.uk
}

\begin{abstract}
Transduction and transmission of an input signal by a neuronal dendrite involves generation, integration and propagation of at least four kinds of information: Chemical concentration such as calcium ions, chemical modification such as phosphorylation cascades, conformational information such as allosteric modulations, and electrical signals such as membrane depolarisation. One cannot claim to understand neuronal function when focussing on a single aspect. However, developing models of the four requires using different formalisms. Furthermore running simulations implies widely different requirements in terms of compute power, storage or results and duration. I will present a few results we obtained about the synaptic function and plasticity in the striatal medium-spiny neuron, using models of signalling networks, allosteric regulations, single particle diffusion and multi-compartment electrical models. I will then discuss how we can sometimes encapsulate the results obtained at a certain level of resolution in order to increase the realism of more abstract models. I will end by outlining how one could envision to build a model striatal neuron that embodies chemical, biochemical and electrical signalling.
\end{abstract}

